\title{
Fractional variational principle of Herglotz for a new class of problems with dependence on the boundaries and a real parameter
}

Cite as: J. Math. Phys. 61, 102701 (2020); https://doi.org/10.1063/5.0021373

Submitted: 09 July 2020 . Accepted: 19 September 2020 . Published Online: 08 October 2020

Ricardo Almeida (D), and Natália Martins (iD)

\section{Journal of}

Mathematical Physics

Young Researcher Award

Recognizing the outstanding work of early career researchers 


\section{Fractional variational principle of Herglotz for a new class of problems with dependence on the boundaries and a real parameter}

Cite as: J. Math. Phys. 61, 102701 (2020); doi: 10.1063/5.0021373

Submitted: 9 July 2020 - Accepted: 19 September 2020 •

Published Online: 8 October 2020

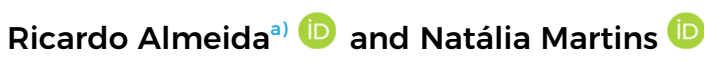

\section{AFFILIATIONS}

Center for Research and Development in Mathematics and Applications (CIDMA), Department of Mathematics,

University of Aveiro, 3810-193 Aveiro, Portugal

a) Author to whom correspondence should be addressed: ricardo.almeida@ua.pt

\section{ABSTRACT}

The fractional variational problem of Herglotz type for the case where the Lagrangian depends on generalized fractional derivatives, the free endpoints conditions, and a real parameter is studied. This type of problem generalizes several problems recently studied in the literature. Moreover, it allows us to unify conservative and non-conservative dynamical processes in the same model. The dependence of the Lagrangian with respect to the boundaries and a free parameter is effective and transforms the standard Herglotz's variational problem into a problem of a different nature.

Published under license by AIP Publishing. https://doi.org/10.1063/5.0021373

\section{INTRODUCTION}

The variational problem of Herglotz was introduced by Herglotz in $1930^{17}$ and constitutes an important generalization of the classical variational problem. In addition, one of the advantages of this type of problems is that the corresponding variational principle, known as Herglotz's variational principle, unifies conservative and non-conservative processes into the same dynamical model. The variational problem proposed by Herglotz can be formulated as follows:

Problem $\left(\mathcal{P}_{\mathcal{H}}\right)$ : Determine trajectories $x \in C^{2}([a, b], \mathbb{R})$ and functional $z \in C^{1}([a, b], \mathbb{R})$ that extremize (maximize or minimize) the value

$$
z(b)
$$

where $(x, z)$ satisfies

$$
\dot{z}(t)=L(t, x(t), \dot{x}(t), z(t)), \quad t \in[a, b]
$$

and

$$
z(a)=\gamma, \quad x(a)=\alpha, \quad x(b)=\beta,
$$

for some fixed $\gamma, \alpha, \beta \in \mathbb{R}$, and the Lagrangian $L$ satisfies given regularity conditions.

It is worth mentioning that if the Lagrangian $L$ does not depend on $z$, problem $\left(\mathcal{P}_{\mathcal{H}}\right)$ reduces to the well-known problem of the calculus of variations,

$$
z(b)=\int_{a}^{b} \tilde{L}(t, x(t), \dot{x}(t)) d t \longrightarrow \text { extr }
$$


where

$$
\tilde{L}(t, x(t), \dot{x}(t))=L(t, x(t), \dot{x}(t))+\frac{\gamma}{b-a} .
$$

Herglotz proved that a necessary condition for a pair $(x, z)$ to be a local extremizer of problem $\left(\mathcal{P}_{\mathcal{H}}\right)$ is given by the following generalized Euler-Lagrange equation: ${ }^{1}$

$$
\begin{array}{r}
\partial_{2} L(t, x(t), \dot{x}(t), z(t))-\frac{d}{d t} \partial_{3} L(t, x(t), \dot{x}(t), z(t)) \\
+\partial_{4} L(t, x(t), \dot{x}(t), z(t)) \cdot \partial_{3} L(t, x(t), \dot{x}(t), z(t))=0, \quad t \in[a, b],
\end{array}
$$

where $\partial_{i} L$ denotes the partial derivative of $L$ with respect to its $i$ th-coordinate. Clearly, if the Lagrangian function $L$ does not depend on the variable $z$, then we get the classical Euler-Lagrange equation (see Ref. 11),

$$
\partial_{2} L(t, x(t), \dot{x}(t))-\frac{d}{d t} \partial_{3} L(t, x(t), \dot{x}(t))=0, \quad t \in[a, b] .
$$

Although the generalized variational problem was introduced in 1930, only after 1996, with the publications s $^{15,16,27}$ of Guenther and his collaborators, it has gained increasing attention from the scientific community. Since then, many important results from the classical calculus of variations have been generalized for the variational problem of Herglotz (see Refs. 1, 3, 13, 14, 22, 34, 35, 37, and 38 and references therein).

In this paper, we focus our attention to variational problems of Herglotz type in the context of fractional calculus. Fractional calculus deals with integrals and derivatives of any positive real order and, therefore, constitutes a generalization of the classical calculus. Many wellknown mathematicians such as Leibniz, Euler, L'Hopital, Riemann, Lioville, and Lacroix worked on the theory of fractional calculus. Although its birth goes back to the end of the XVIIth century, fractional calculus has achieved remarkable progress only in the last decades, mainly with the applicability of such operators in diverse research areas, such as physics, mechanics, biology, medicine, and economics. ${ }^{12,19,21,28,36}$

In the literature, there are many different definitions of fractional derivatives. The Riemann-Liouville, the Caputo, the Hadamard, and the Erdélyi-Kober derivatives are some of the most important ones. In this paper, we consider two recent notions of fractional derivatives that generalize the ones mentioned above: the Caputo and Riemann-Liouville fractional derivatives with respect to another function (see Refs. 5 and 32 , respectively).

The fractional calculus of variations, which combines the calculus of variations and fractional calculus, was initiated in $1996^{29,30}$ and has gained a lot of attention from the scientific comunity because it allows a more accurate description of some physical phenomena. In these two papers, F. Riewe obtained a version of the Euler-Lagrange equations for problems of the calculus of variations with fractional derivatives, combining the conservative and non-conservative cases. Since then, the study of fractional calculus of variations is a subject of current strong research (see, e.g., Refs. 2, 4, 6, 20, and 24 and references therein). For an overview on the subject, we refer to the recent books. ${ }^{8,23,25}$

Based on the aspects as stated above, our motivation is to study the variational problems of Herglotz type for problems where the Lagrangian depends on fractional derivatives with respect to another function. Moreover, we will consider the more general case in which the Lagrangian also depends on the free endpoint conditions and a real parameter, creating what we call non-standard fractional Herglotz's variational problems. This new type of generalized variational problems allows us to obtain new results that generalize several important results recently published in the literature. For the importance of the Lagrangian dependence of a real parameter and the free endpoint conditions, we refer to physical problems ${ }^{18}$ and to economics problems, ${ }^{39}$ respectively. This dependency is effective and transforms the standard fractional Herglotz's variational problem ${ }^{3,37}$ into a problem of a different nature.

This paper is structured as follows. In Sec. II, we recall the basic definitions and results from fractional calculus that are needed in the paper. In Sec. III, we present our main results. First, we prove the fractional variational principle for non-standard problems of Herglotz type, with and without fixed boundary conditions. Then, we generalize this variational principle for problems with time delay, with higher-order fractional derivatives, and with several independent variables. We finalize our work with two illustrative examples.

\section{PRELIMINARIES}

For the convenience of the reader, we recall some basic concepts and preliminary results on fractional calculus, needed in the sequel. For a comprehensive knowledge of fractional calculus, we refer the reader to the books. ${ }^{31,32}$ As usual, $\Gamma$ represents the Gamma function, and $[\alpha]$ denotes the integer part of $\alpha \in \mathbb{R}$.

Definition 2.1 (Riemann-Liouville fractional integrals with respect to another function). ${ }^{32}$ Let $\alpha \in \mathbb{R}^{+}, \psi \in C^{1}([a, b], \mathbb{R})$ be a function such that $\dot{\psi}(t)>0$, for all $t \in[a, b]$, and $x:[a, b] \rightarrow \mathbb{R}$ be an integrable function. The left and right sided Riemann-Liouville fractional integrals of $x$ with respect to $\psi$, of order $\alpha$, are defined as

$$
\left(I_{a^{+}}^{\alpha, \psi} x\right)(t):=\frac{1}{\Gamma(\alpha)} \int_{a}^{t} \dot{\psi}(\tau)(\psi(t)-\psi(\tau))^{\alpha-1} x(\tau) d \tau, \quad t>a,
$$


and

$$
\left(I_{b^{-}}^{\alpha, \psi} x\right)(t):=\frac{1}{\Gamma(\alpha)} \int_{t}^{b} \dot{\psi}(\tau)(\psi(\tau)-\psi(t))^{\alpha-1} x(\tau) d \tau, \quad t<b,
$$

respectively.

We remark that under some additional assumptions,

$$
I_{a^{+}}^{\alpha, \psi} x=I_{b^{-}}^{\alpha, \psi} x=x, \quad \text { as } \quad \alpha \rightarrow 0^{+} .
$$

It is worth mentioning that the Riemann-Liouville, the Hadamard, and the Erdélyi-Kober fractional integrals are obtained when we consider $\psi(t)=t, \psi(t)=\ln (t)$, and $\psi(t)=t^{\sigma}(\sigma>0)$, respectively.

Definition 2.2 (Riemann-Liouville fractional derivatives with respect to another function). ${ }^{32}$ Let $\alpha \in \mathbb{R}^{+}, \psi \in C^{n}([a, b], \mathbb{R})$ be a function such that $\dot{\psi}(t)>0$, for all $t \in[a, b]$, and $x:[a, b] \rightarrow \mathbb{R}$ be an integrable function. The left and right sided Riemann-Liouville fractional derivatives of $x$ with respect to $\psi$, of order $\alpha$, are given by the formulas

$$
\left(D_{a^{+}}^{\alpha, \psi} x\right)(t):=\left(\frac{1}{\dot{\psi}(t)} \frac{d}{d t}\right)^{n}\left(I_{a^{+}}^{n-\alpha, \psi} x\right)(t), \quad t>a,
$$

and

$$
\left(D_{b^{-}}^{\alpha, \psi} x\right)(t):=\left(-\frac{1}{\dot{\psi}(t)} \frac{d}{d t}\right)^{n}\left(I_{b^{-}}^{n-\alpha, \psi} x\right)(t), \quad t<b,
$$

where $n=[\alpha]+1$.

If we interchange the order of the classical derivative with the fractional integral, we obtain the definition of the Caputo fractional derivatives with respect to another function.

Definition 2.3 (Caputo fractional derivatives with respect to another function). ${ }^{5}$ Let $\alpha \in \mathbb{R}^{+}$and $n \in \mathbb{N}$ be defined by $n=[\alpha]+1$ if $\alpha \notin \mathbb{N}$ and $n=\alpha$ if $\alpha \in \mathbb{N}$. Given two functions $x, \psi \in C^{n}([a, b], \mathbb{R})$, with $\dot{\psi}(t)>0$, for all $t \in[a, b]$, the left and right sided Caputo fractional derivatives of $x$ with respect to $\psi$, of order $\alpha$, are defined as

$$
\left({ }^{C} D_{a^{+}}^{\alpha, \psi} x\right)(t):=\left(I_{a^{+}}^{n-\alpha, \psi}\left(\frac{1}{\dot{\psi}(t)} \frac{d}{d t}\right)^{n} x\right)(t), \quad t>a,
$$

and

$$
\left({ }^{C} D_{b^{-}}^{\alpha, \psi} x\right)(t):=\left(I_{b^{-}}^{n-\alpha, \psi}\left(-\frac{1}{\dot{\psi}(t)} \frac{d}{d t}\right)^{n} x\right)(t), \quad t<b,
$$

respectively.

Remark 1. We remark that

1. if $\alpha=m \in \mathbb{N}$, then $\left({ }^{C} D_{a^{+}}^{\alpha, \psi} x\right)(t)=\left(D_{a^{+}}^{\alpha, \psi} x\right)(t)=\left(\frac{1}{\dot{\psi}(t)} \frac{d}{d t}\right)^{m} x(t)$ and $\left({ }^{C} D_{b^{-}}^{\alpha, \psi} x\right)(t)=\left(D_{b^{-}}^{\alpha, \psi} x\right)(t)=\left(-\frac{1}{\dot{\psi}(t)} \frac{d}{d t}\right)^{m} x(t)$;

2. if $\alpha \in \mathbb{R}^{+} \backslash \mathbb{N}$, then

$$
\left(D_{a^{+}}^{\alpha, \psi} x\right)(t)=\frac{1}{\Gamma(n-\alpha)}\left(\frac{1}{\dot{\psi}(t)} \frac{d}{d t}\right)^{n} \int_{a}^{t} \dot{\psi}(\tau)(\psi(t)-\psi(\tau))^{n-\alpha-1} x(\tau) d \tau
$$

and

$$
\left(D_{b^{-}}^{\alpha, \psi} x\right)(t)=\frac{1}{\Gamma(n-\alpha)}\left(-\frac{1}{\dot{\psi}(t)} \frac{d}{d t}\right)^{n} \int_{t}^{b} \dot{\psi}(\tau)(\psi(\tau)-\psi(t))^{n-\alpha-1} x(\tau) d \tau
$$

3. if $\alpha \in \mathbb{R}^{+} \backslash \mathbb{N}$, then

$$
\left({ }^{C} D_{a^{+}}^{\alpha, \psi} x\right)(t)=\frac{1}{\Gamma(n-\alpha)} \int_{a}^{t} \dot{\psi}(\tau)(\psi(t)-\psi(\tau))^{n-\alpha-1}\left(\frac{1}{\dot{\psi}(\tau)} \frac{d}{d \tau}\right)^{n} x(\tau) d \tau
$$


and

$$
\left({ }^{C} D_{b^{-}}^{\alpha, \psi} x\right)(t)=\frac{1}{\Gamma(n-\alpha)} \int_{t}^{b} \dot{\psi}(\tau)(\psi(\tau)-\psi(t))^{n-\alpha-1}\left(-\frac{1}{\dot{\psi}(\tau)} \frac{d}{d \tau}\right)^{n} x(\tau) d \tau ;
$$

4. the Riemann-Liouville, the Hadamard, and the Erdélyi-Kober fractional derivatives are obtained from the operators $D_{a^{+}}^{\alpha, \psi} x$ and $D_{b^{-}}^{\alpha, \psi} x$ when we consider $\psi(t)=t, \psi(t)=\ln (t)$, and $\psi(t)=t^{\sigma}(\sigma>0)$, respectively, while the right and left Caputo derivatives are obtained from ${ }^{C} D_{a^{+}}^{\alpha, \psi} x$ and ${ }^{C} D_{b^{-}}^{\alpha, \psi} x$ when $\psi(t)=t$, respectively.

To finalize this section, we present the integration by parts formula that plays a crucial role in deriving the generalized Euler-Lagrange equations. For more details on $\psi$-Caputo fractional derivatives, we refer to Ref. 5.

Theorem 2.4. (Ref. 5). Let $x \in C([a, b], \mathbb{R})$ and $y, \psi \in C^{n}([a, b], \mathbb{R})$ be two functions, with $\dot{\psi}(t)>0$, for all $t \in[a, b]$. Then,

$$
\begin{aligned}
\int_{a}^{b} x(t) & \cdot\left({ }^{C} D_{a^{+}}^{\alpha, \psi} y\right)(t) d t=\int_{a}^{b} y(t) \cdot\left(D_{b^{-}}^{\alpha, \psi} \frac{x}{\dot{\psi}}\right)(t) \dot{\psi}(t) d t \\
+ & +\left[\sum_{k=0}^{n-1}\left(-\frac{1}{\dot{\psi}(t)} \frac{d}{d t}\right)^{k}\left(I_{b^{-}}^{n-\alpha, \psi} \frac{x}{\dot{\psi}}\right)(t) \cdot\left(\frac{1}{\dot{\psi}(t)} \frac{d}{d t}\right)^{n-k-1} y(t)\right]_{t=a}^{t=b}
\end{aligned}
$$

and

$$
\begin{aligned}
\int_{a}^{b} x(t) & \cdot\left({ }^{C} D_{b^{-}}^{\alpha, \psi} y\right)(t) d t=\int_{a}^{b} y(t) \cdot\left(D_{a^{+}}^{\alpha, \psi} \frac{x}{\dot{\psi}}\right)(t) \dot{\psi}(t) d t \\
+ & +\left[\sum_{k=0}^{n-1}(-1)^{n-k}\left(\frac{1}{\dot{\psi}(t)} \frac{d}{d t}\right)^{k}\left(I_{a^{+}}^{n-\alpha, \psi} \frac{x}{\dot{\psi}}\right)(t) \cdot\left(\frac{1}{\dot{\psi}(t)} \frac{d}{d t}\right)^{n-k-1} y(t)\right]_{t=a}^{t=b} .
\end{aligned}
$$

\section{MAIN RESULTS}

In this section, we prove necessary optimality conditions for several variational problems of Herglotz type. For simplicity of presentation, we limit ourselves to the scalar case since it is obvious how to generalize all of our results for the case of vector functions $x:[a, b] \rightarrow \mathbb{R}^{n}$. From now on, let $\alpha, \beta \in] 0,1[$.

\section{A. Herglotz fractional variational principle}

Let us consider the following fractional variational problem. In order to simplify expressions, we will use the notations

$$
\begin{aligned}
{[x, z, \zeta](t) } & :=\left(t, x(t),\left({ }^{C} D_{a^{+}}^{\alpha, \psi} x\right)(t),\left({ }^{C} D_{b^{-}}^{\beta, \psi} x\right)(t), z(t), x(a), x(b), \zeta\right), \\
{[x, \zeta](t) } & :=\left(t, x(t),\left({ }^{C} D_{a^{+}}^{\alpha, \psi} x\right)(t),\left({ }^{C} D_{b^{-}}^{\beta, \psi} x\right)(t), x(a), x(b), \zeta\right) .
\end{aligned}
$$

Problem $\left(\mathcal{P}_{1}\right)$ : Determine $x \in C^{1}([a, b], \mathbb{R}), z \in C^{1}([a, b], \mathbb{R})$, and $\zeta \in \mathbb{R}$ that extremize

$$
z(b)
$$

where $(x, z, \zeta)$ satisfies the differential equation

$$
\dot{z}(t)=L[x, z, \zeta](t), \quad t \in[a, b]
$$

and

$$
z(a)=\gamma
$$

for a given $\gamma \in \mathbb{R}$. We assume that ${ }^{C} D_{a^{+}}^{\alpha, \psi} x,{ }^{C} D_{b^{-}}^{\beta, \psi} x \in C^{1}([a, b], \mathbb{R})$ and $L$ satisfies the following conditions:

1. $L:[a, b] \times \mathbb{R}^{7} \rightarrow \mathbb{R}$ is of class $C^{1}$;

2. functions $t \rightarrow \frac{\lambda(t) \cdot \partial_{3} L[x, z, \zeta](t)}{\dot{\psi}(t)}$ and $t \rightarrow \frac{\lambda(t) \cdot \partial_{4} L[x, z, \zeta](t)}{\dot{\psi}(t)}$ are such that

$$
D_{b^{-}}^{\alpha, \psi}\left(\frac{\lambda \cdot \partial_{3} L[x, z, \zeta]}{\dot{\psi}}\right) \text { and } D_{a^{+}}^{\beta, \psi}\left(\frac{\lambda \cdot \partial_{4} L[x, z, \zeta]}{\dot{\psi}}\right)
$$


exist and are continuous on $[a, b]$, for all admissible triplet $(x, z, \zeta)$, where

$$
\lambda(t):=e^{-\int_{a}^{t} \partial_{5} L[x, z, \zeta](s) d s}, \quad t \in[a, b] .
$$

Remark 2. We remark that

1. from (3), it follows that $z$ depends on $t, x$, and $\zeta$, but to make the notation simpler, we write $z(t)$ instead of $z[x, \zeta](t)$;

2. if the Lagrangian $L$ does not depend on $z$, like in the classical variational problem, $\lambda(t)=1$ for all $t \in[a, b]$.

Theorem 3.1 (Non-standard Herglotz's fractional variational principle and natural boundary conditions). If ( $x, z$, $\zeta)$ is a solution of problem $\left(\mathcal{P}_{1}\right)$, then $(x, z, \zeta)$ satisfies the fractional Euler-Lagrange equation

$$
\lambda(t) \cdot \partial_{2} L[x, z, \zeta](t)+D_{b^{-}}^{\alpha, \psi}\left(\frac{\lambda \cdot \partial_{3} L[x, z, \zeta]}{\dot{\psi}}\right)(t) \cdot \dot{\psi}(t)+D_{a^{+}}^{\beta, \psi}\left(\frac{\lambda \cdot \partial_{4} L[x, z, \zeta]}{\dot{\psi}}\right)(t) \cdot \dot{\psi}(t)=0,
$$

for all $t \in[a, b]$ and

$$
\int_{a}^{b} \lambda(t) \cdot \partial_{8} L[x, z, \zeta](t) d t=0
$$

Moreover,

1. if $x(a)$ is free, then $(x, z, \zeta)$ satisfies the following condition:

$$
\int_{a}^{b} \lambda(t) \cdot \partial_{6} L[x, z, \zeta](t) d t=\left(I_{b^{-}}^{1-\alpha, \psi} \frac{\lambda \cdot \partial_{3} L[x, z, \zeta]}{\dot{\psi}}\right)(a)-\left(I_{a^{+}}^{1-\beta, \psi} \frac{\lambda \cdot \partial_{4} L[x, z, \zeta]}{\dot{\psi}}\right)(a),
$$

2. if $x(b)$ is free, then $(x, z, \zeta)$ satisfies the following condition:

$$
\int_{a}^{b} \lambda(t) \cdot \partial_{7} L[x, z, \zeta](t) d t=\left(I_{a^{+}}^{1-\beta, \psi} \frac{\lambda \cdot \partial_{4} L[x, z, \zeta]}{\dot{\psi}}\right)(b)-\left(I_{b^{-}}^{1-\alpha, \psi} \frac{\lambda \cdot \partial_{3} L[x, z, \zeta]}{\dot{\psi}}\right)(b) .
$$

Proof. Suppose that $(x, z, \zeta)$ is a solution of problem $\left(\mathcal{P}_{1}\right)$, and let $\eta \in C^{1}([a, b], \mathbb{R})$, such that its Caputo fractional derivatives are continuously differentiable, and $\Delta \zeta \in \mathbb{R}$ be arbitrary. Define $\varphi:[a, b] \rightarrow \mathbb{R}$ by

$$
\varphi(t)=\left.\frac{d}{d \varepsilon} z[x+\varepsilon \eta, \zeta+\varepsilon \Delta \zeta](t)\right|_{\varepsilon=0} .
$$

Since $z(a)$ is fixed, it is clear that $\varphi(a)=0$. Define

$$
f(\varepsilon)=z[x+\varepsilon \eta, \zeta+\varepsilon \Delta \zeta](b) .
$$

Since $z(b)$ is a local extremum, we conclude that 0 is a local extremizer of $f$ and, therefore,

$$
\varphi(b)=\left.\frac{d}{d \varepsilon} z[x+\varepsilon \eta, \zeta+\varepsilon \Delta \zeta](b)\right|_{\varepsilon=0}=\dot{f}(0)=0 .
$$

Observe that

$$
\begin{aligned}
\dot{\varphi}(t)= & \left.\frac{d}{d t} \frac{d}{d \varepsilon} z[x+\varepsilon \eta, \zeta+\varepsilon \Delta \zeta](t)\right|_{\varepsilon=0} \\
= & \left.\frac{d}{d \varepsilon} \frac{d}{d t} z[x+\varepsilon \eta, \zeta+\varepsilon \Delta \zeta](t)\right|_{\varepsilon=0} \\
= & \partial_{2} L[x, z, \zeta](t) \cdot \eta(t)+\partial_{3} L[x, z, \zeta](t) \cdot\left({ }^{C} D_{a^{+}}^{\alpha, \psi} \eta\right)(t)+\partial_{4} L[x, z, \zeta](t) \cdot\left({ }^{C} D_{b^{-}}^{\beta, \psi} \eta\right)(t) \\
& +\left.\partial_{5} L[x, z, \zeta](t) \cdot \frac{d}{d \varepsilon} z[x+\varepsilon \eta, \zeta+\varepsilon \Delta \zeta](t)\right|_{\varepsilon=0}+\partial_{6} L[x, z, \zeta](t) \cdot \eta(a) \\
& +\partial_{7} L[x, z, \zeta](t) \cdot \eta(b)+\partial_{8} L[x, z, \zeta](t) \cdot \Delta \zeta \\
= & \partial_{2} L[x, z, \zeta](t) \cdot \eta(t)+\partial_{3} L[x, z, \zeta](t) \cdot\left({ }^{C} D_{a^{+}}^{\alpha, \psi} \eta\right)(t)+\partial_{4} L[x, z, \zeta](t) \cdot\left({ }^{C} D_{b^{-}}^{\beta, \psi} \eta\right)(t) \\
& +\partial_{5} L[x, z, \zeta](t) \cdot \varphi(t)+\partial_{6} L[x, z, \zeta](t) \cdot \eta(a)+\partial_{7} L[x, z, \zeta](t) \cdot \eta(b)+\partial_{8} L[x, z, \zeta](t) \cdot \Delta \zeta,
\end{aligned}
$$


which is equivalent to

$$
\begin{aligned}
& \dot{\varphi}(t)-\partial_{5} L[x, z, \zeta](t) \cdot \varphi(t) \\
& =\partial_{2} L[x, z, \zeta](t) \cdot \eta(t)+\partial_{3} L[x, z, \zeta](t) \cdot\left({ }^{C} D_{a^{+}}^{\alpha, \psi} \eta\right)(t)+\partial_{4} L[x, z, \zeta](t) \cdot\left({ }^{C} D_{b^{-}}^{\beta, \psi} \eta\right)(t) \\
& +\partial_{6} L[x, z, \zeta](t) \cdot \eta(a)+\partial_{7} L[x, z, \zeta](t) \cdot \eta(b)+\partial_{8} L[x, z, \zeta](t) \cdot \Delta \zeta .
\end{aligned}
$$

The solution of this first-order linear differential equation is given by

$$
\begin{aligned}
& e^{-\int_{a}^{t} \partial_{5} L[x, z, \zeta](s) d s} \varphi(t)-\varphi(a) \\
& =\int_{a}^{t} e^{-\int_{a}^{s} \partial_{5} L[x, z, \zeta](\tau) d \tau} \cdot\left(\partial_{2} L[x, z, \zeta](s) \cdot \eta(s)+\partial_{3} L[x, z, \zeta](s) \cdot\left({ }^{C} D_{a^{+}}^{\alpha, \psi} \eta\right)(s)\right. \\
& +\partial_{4} L[x, z, \zeta](s) \cdot\left({ }^{C} D_{b^{-}}^{\beta, \psi} \eta\right)(s)+\partial_{6} L[x, z, \zeta](s) \cdot \eta(a) \\
& \left.+\partial_{7} L[x, z, \zeta](s) \cdot \eta(b)+\partial_{8} L[x, z, \zeta](s) \cdot \Delta \zeta\right) d s
\end{aligned}
$$

Considering $t=b$ in Eq. (11), we get

$$
\begin{aligned}
& \int_{a}^{b} \lambda(s) \cdot\left(\partial_{2} L[x, z, \zeta](s) \cdot \eta(s)+\partial_{3} L[x, z, \zeta](s) \cdot\left({ }^{C} D_{a^{+}}^{\alpha, \psi} \eta\right)(s)+\partial_{4} L[x, z, \zeta](s) \cdot\left({ }^{C} D_{b^{-}}^{\beta, \psi} \eta\right)(s)\right. \\
& \left.+\partial_{6} L[x, z, \zeta](s) \cdot \eta(a)+\partial_{7} L[x, z, \zeta](s) \cdot \eta(b)+\partial_{8} L[x, z, \zeta](s) \cdot \Delta \zeta\right) d s=0
\end{aligned}
$$

because $\varphi(a)=\varphi(b)=0$. Using fractional integration by parts (see Theorem 2.4), we get

$$
\begin{aligned}
& \int_{a}^{b}\left(\lambda(s) \cdot \partial_{2} L[x, z, \zeta](s)+D_{b^{-}}^{\alpha, \psi}\left(\frac{\lambda \cdot \partial_{3} L[x, z, \zeta]}{\dot{\psi}}\right)(s) \cdot \dot{\psi}(s)\right. \\
& \left.+D_{a^{+}}^{\beta, \psi}\left(\frac{\lambda \cdot \partial_{4} L[x, z, \zeta]}{\dot{\psi}}\right)(s) \cdot \dot{\psi}(s)\right) \eta(s) d s \\
& +\left[\left(I_{b^{-}}^{1-\alpha, \psi} \frac{\lambda \cdot \partial_{3} L[x, z, \zeta]}{\dot{\psi}}\right)(s) \cdot \eta(s)\right]_{s=a}^{s=b}-\left[\left(I_{a^{+}}^{1-\beta, \psi} \frac{\lambda \cdot \partial_{4} L[x, z, \zeta]}{\dot{\psi}}\right)(s) \cdot \eta(s)\right]_{s=a}^{s=b} \\
& +\int_{a}^{b} \lambda(s) \cdot\left(\partial_{6} L[x, z, \zeta](s) \cdot \eta(a)+\partial_{7} L[x, z, \zeta](s) \cdot \eta(b)+\partial_{8} L[x, z, \zeta](s) \cdot \Delta \zeta\right) d s=0 .
\end{aligned}
$$

Since Eq. (12) holds for all admissible variations $\eta$ and all $\Delta \zeta \in \mathbb{R}$, then, in particular, it holds for all functions $\eta$ such that $\eta(a)=\eta(b)=0$ and for $\Delta \zeta=0$, proving that

$$
\begin{aligned}
& \int_{a}^{b}\left(\lambda(s) \cdot \partial_{2} L[x, z, \zeta](s)+D_{b^{-}}^{\alpha, \psi}\left(\frac{\lambda \cdot \partial_{3} L[x, z, \zeta]}{\dot{\psi}}\right)(s) \cdot \dot{\psi}(s)\right. \\
& \left.+D_{a^{+}}^{\beta, \psi}\left(\frac{\lambda \cdot \partial_{4} L[x, z, \zeta]}{\dot{\psi}}\right)(s) \cdot \dot{\psi}(s)\right) \eta(s) d s=0 .
\end{aligned}
$$

From the arbitrariness of $\eta$, applying the fundamental lemma of the calculus of variations, we obtain (6). Restricting $\eta$ to be null, we get from (12) that

$$
\int_{a}^{b} \lambda(s) \cdot \partial_{8} L[x, z, \zeta](s) \cdot \Delta \zeta d s=0
$$

and by the arbitrariness of $\Delta \zeta$, we deduce (7).

Next, we prove the natural boundary conditions (8) and (9). To prove (8), substitute (6) and (7) into (12) and consider $\eta$ such that $\eta(a) \neq 0$ and $\eta(b)=0$. To prove (9), substitute (6) and (7) into (12) and consider $\eta$ such that $\eta(a)=0$ and $\eta(b) \neq 0$. This concludes the proof.

From Theorem 3.1 we can obtain several important corollaries. For example, if the Lagrangian $L$ does not depends on $z$, we get the following result:

Corollary 1 (Ref. 9). If the pair $(x, \zeta)$ is a local extremizer of functional 


$$
\mathcal{J}(x, \zeta):=\int_{a}^{b} L\left(t, x(t),\left({ }^{C} D_{a^{+}}^{\alpha, \psi} x\right)(t),\left({ }^{C} D_{b^{-}}^{\beta, \psi} x\right)(t), x(a), x(b), \zeta\right)(t) d t
$$

and if the maps

$$
t \mapsto\left(D_{b^{-}}^{\alpha, \psi} \frac{\partial_{3} L[x, \zeta]}{\dot{\psi}}\right)(t) \quad \text { and } \quad t \mapsto\left(D_{a^{+}}^{\beta, \psi} \frac{\partial_{4} L[x, \zeta]}{\dot{\psi}}\right)(t)
$$

are continuous for all $t \in[a, b]$, then

$$
\partial_{2} L[x, \zeta](t)+\left(D_{b^{-}}^{\alpha, \psi} \frac{\partial_{3} L[x, \zeta]}{\dot{\psi}}\right)(t) \cdot \dot{\psi}(t)+\left(D_{a^{+}}^{\beta, \psi} \frac{\partial_{4} L[x, \zeta]}{\dot{\psi}}\right)(t) \cdot \dot{\psi}(t)=0, \quad t \in[a, b],
$$

and

$$
\int_{a}^{b} \partial_{7} L[x, \zeta](t) d t=0
$$

Moreover,

1. if $x(a)$ is free, then

2. if $x(b)$ is free, then

$$
\int_{a}^{b} \partial_{5} L[x, \zeta](t) d t=\left(I_{b^{-}}^{1-\alpha, \psi} \frac{\partial_{3} L[x, \zeta]}{\dot{\psi}}\right)(a)-\left(I_{a^{+}}^{1-\beta, \psi} \frac{\partial_{4} L[x, \zeta]}{\dot{\psi}}\right)(a)
$$

$$
\int_{a}^{b} \partial_{6} L[x, \zeta](t) d t=\left(I_{a^{+}}^{1-\beta, \psi} \frac{\partial_{4} L[x, \zeta]}{\dot{\psi}}\right)(b)-\left(I_{b^{-}}^{1-\alpha, \psi} \frac{\partial_{3} L[x, \zeta]}{\dot{\psi}}\right)(b) .
$$

In the particular case where the kernel $\psi$ is the identity, $\alpha \rightarrow 1^{-}$and $\beta \rightarrow 1^{-}$; then, problem $\left(\mathcal{P}_{1}\right)$ reduces to the classical non-standard Herglotz's variational problem recently studied in Ref. 26.

Corollary 2. (Ref. 26). If $(x, z, \zeta)$ is a solution of the non-standard Herglotz's variational problem

$$
z(b) \rightarrow \text { extr }
$$

where $(x, z, \zeta)$ is such that

$$
\dot{z}(t)=L(t, x(t), \dot{x}(t), z(t), x(a), x(b), \zeta), \quad t \in[a, b],
$$

and $z(a)=\gamma$, for a fixed $\gamma \in \mathbb{R}$, then the following conditions holds:

$$
\partial_{2} L[x, z, \zeta](t)+\partial_{4} L[x, z, \zeta](t) \cdot \partial_{3} L[x, z, \zeta](t)-\frac{d}{d t} \partial_{3} L[x, z, \zeta](t)=0, \quad t \in[a, b]
$$

and

$$
\int_{a}^{b} \lambda(t) \cdot \partial_{7} L[x, z, \zeta](t) d t=0
$$

where $\lambda(t)=e^{-\int_{a}^{t} \partial_{4} L[x, z, \zeta](s) d s}$. Moreover,

1. if $x(a)$ is free, then $\partial_{3} L[x, z, \zeta](a)=\frac{1}{\lambda(a)} \int_{a}^{b} \lambda(t) \cdot \partial_{5} L[x, z, \zeta] d t$;

2. if $x(b)$ is free, then $\partial_{3} L[x, z, \zeta](b)=-\frac{1}{\lambda(b)} \int_{a}^{b} \lambda(t) \cdot \partial_{6} L[x, z, \zeta] d t$.

Remark 3. It is also important to remark that

1. if $\psi$ is the identity and the Lagrangian function $L$ does not depends on $\left({ }^{C} D_{b^{-}}^{\beta, \psi} x\right)(t), x(a), x(b)$, and $\zeta$, then Theorem 3.1 reduces to Theorem 3.9 of Ref. 3. Comparing the natural boundary conditions given in Theorem 3.1 with the one given in Theorem 3.9 of Ref. 3 , we may conclude that the standard and non-standard Herglotz's variational problems are not equivalent;

2. if $\psi$ is the identity, $\alpha \rightarrow 1^{-}$and $\beta \rightarrow 1^{-}$, and if the Lagrangian does not depend on the state values $x(a)$ and $x(b)$, and on the parameter $\zeta$, then we obtain the generalized Euler-Lagrange equation (1) and the natural boundary conditions $\partial_{3} L(a, x(a), \dot{x}(a), z(a))=0$ and $\partial_{3} L(b, x(b), \dot{x}(b), z(b))=0$ proved in Ref. 33 ; 
3. if $\psi$ is the identity, $\alpha \rightarrow 1^{-}$and $\beta \rightarrow 1^{-}$, and $L$ is independent of $z, x(a), x(b)$, and $\zeta$, problem $\left(\mathcal{P}_{1}\right)$ reduces to the fundamental problem of the calculus of variations. Hence, the generalized Euler-Lagrange equation (6) reduces to the classical Euler-Lagrange equation (2), and conditions (8) and (9) reduce to the well-known natural boundary conditions $\partial_{3} L(a, x(a), \dot{x}(a))=0$ and $\partial_{3} L(b, x(b), \dot{x}(b))=0($ see, e.g., Ref. 11).

\section{B. Herglotz fractional variational principle with time delay}

Inspired by the fact that variational systems with time delay play an important role in the modeling of phenomena in various applied fields, we consider now the following generalized variational problem. For simplicity of notation, we use the notation

$$
\begin{aligned}
{[x, z, \zeta]_{\tau}(t) } & :=\left(t, x(t), x(t-\tau),\left({ }^{C} D_{a^{+}}^{\alpha, \psi} x\right)(t),\left({ }^{C} D_{b^{-}}^{\beta, \psi} x\right)(t), z(t), x(a), x(b), \zeta\right), \\
{[x, \zeta]_{\tau}(t) } & :=\left(t, x(t), x(t-\tau),\left({ }^{C} D_{a^{+}}^{\alpha, \psi} x\right)(t),\left({ }^{C} D_{b^{-}}^{\beta, \psi} x\right)(t), x(a), x(b), \zeta\right) .
\end{aligned}
$$

Problem $\left(\mathcal{P}_{2}\right)$ : Let $a<b$ and $\tau$ be a fixed real number such that $0 \leq \tau<b-a$. Determine $x \in C^{1}([a-\tau, b], \mathbb{R}), z \in C^{1}([a, b], \mathbb{R})$, and $\zeta \in \mathbb{R}$ that extremize

$$
z(b)
$$

where $x(t)=\delta(t), t \in[a-\tau, a], \delta \in C^{1}([a-\tau, a], \mathbb{R})$ is a given initial function,

$$
\dot{z}(t)=L[x, z, \zeta]_{\tau}(t), \quad t \in[a, b],
$$

and

$$
z(a)=\gamma
$$

for a given $\gamma \in \mathbb{R}$. We assume that ${ }^{C} D_{a^{+}}^{\alpha, \psi} x,{ }^{C} D_{b^{-}}^{\beta, \psi} x \in C^{1}([a, b], \mathbb{R})$ and $L$ satisfies the following conditions:

1. $L:[a, b] \times \mathbb{R}^{8} \rightarrow \mathbb{R}$ is of class $C^{1}$;

2. there exist and are continuous the functions

$$
t \mapsto\left(D_{(b-\tau)^{-}}^{\alpha, \psi} \frac{\lambda . \partial_{4} L[x, z, \zeta]_{\tau}}{\dot{\psi}}\right)(t) \quad \text { and } \quad t \mapsto\left(D_{a^{+}}^{\beta, \psi} \frac{\lambda . \partial_{5} L[x, z, \zeta]_{\tau}}{\dot{\psi}}\right)(t) \quad \text { on } \quad[a, b-\tau]
$$

and

$$
t \mapsto\left(D_{b^{-}}^{\alpha, \psi} \frac{\lambda . \partial_{4} L[x, z, \zeta]_{\tau}}{\dot{\psi}}\right)(t) \quad \text { and } \quad t \mapsto\left(D_{(b-\tau)^{+}}^{\beta, \psi} \frac{\lambda . \partial_{5} L[x, z, \zeta]_{\tau}}{\dot{\psi}}\right)(t) \quad \text { on } \quad[b-\tau, b]
$$

where

$$
\lambda(t):=e^{-\int_{a}^{t} \partial_{6} L[x, z, \zeta]_{\tau}(s) d s}, \quad t \in[a, b] .
$$

Theorem 3.2 (Non-standard Herglotz fractional variational principle with time delay and natural boundary condition). If ( $x, z, \zeta)$ is $a$ solution of problem $\left(\mathcal{P}_{2}\right)$, then $(x, z, \zeta)$ satisfies the following fractional Euler-Lagrange equations:

$$
\begin{gathered}
\lambda(t) \cdot \partial_{2} L[x, z, \zeta]_{\tau}(t)+\lambda(t+\tau) \cdot \partial_{3} L[x, z, \zeta]_{\tau}(t+\tau) \\
+\left(D_{(b-\tau)^{-}}^{\alpha, \psi} \frac{\lambda \cdot \partial_{4} L[x, z, \zeta]_{\tau}}{\dot{\psi}}\right)(t) \cdot \dot{\psi}(t)+\left(D_{a^{+}}^{\beta \psi} \frac{\lambda \cdot \partial_{5} L[x, z, \zeta]_{\tau}}{\dot{\psi}}\right)(t) \cdot \dot{\psi}(t) \\
\quad-\frac{1}{\Gamma(1-\alpha)} \frac{d}{d t} \int_{b-\tau}^{b} \lambda(s)(\psi(s)-\psi(t))^{-\alpha} \cdot \partial_{4} L[x, z, \zeta]_{\tau}(s) d s=0,
\end{gathered}
$$

for all $t \in[a, b-\tau]$, and

$$
\begin{aligned}
\lambda(t) & \cdot \partial_{2} L[x, z, \zeta]_{\tau}(t)+\left(D_{b^{-}}^{\alpha, \psi} \frac{\lambda \cdot \partial_{4} L[x, z, \zeta]_{\tau}}{\dot{\psi}}\right)(t) \cdot \dot{\psi}(t)+\left(D_{(b-\tau)^{+}}^{\beta, \psi} \frac{\lambda \cdot \partial_{5} L[x, z, \zeta]_{\tau}}{\dot{\psi}}\right)(t) \cdot \dot{\psi}(t) \\
& +\frac{1}{\Gamma(1-\beta)} \frac{d}{d t} \int_{a}^{b-\tau} \lambda(s)(\psi(t)-\psi(s))^{-\beta} \cdot \partial_{5} L[x, z, \zeta]_{\tau}(s) d s=0
\end{aligned}
$$


for all $t \in[b-\tau, b]$. Moreover,

$$
\int_{a}^{b} \lambda(t) \cdot \partial_{9} L[x, z, \zeta]_{\tau}(t) d t=0
$$

and if $x(b)$ is free, then

$$
\int_{a}^{b} \lambda(t) \cdot \partial_{8} L[x, z, \zeta]_{\tau}(t) d t=\left(I_{a^{+}}^{1-\beta, \psi} \frac{\lambda \cdot \partial_{5} L[x, z, \zeta]_{\tau}}{\dot{\psi}}\right)(b)-\left(I_{b^{-}}^{1-\alpha, \psi} \frac{\lambda \cdot \partial_{4} L[x, z, \zeta]_{\tau}}{\dot{\psi}}\right)(b)
$$

Proof. Let $\eta \in C^{1}([a-\tau, b], \mathbb{R})$ be such that $\eta(t)=0$, for all $t \in[a-\tau, a]$, and its Caputo fractional derivatives are continuously differentiable, and let $\Delta \zeta$ be an arbitrary fixed real. Define $\varphi:[a, b] \rightarrow \mathbb{R}$ by

$$
\varphi(t)=\left.\frac{d}{d \varepsilon} z[x+\varepsilon \eta, \zeta+\varepsilon \Delta \zeta]_{\tau}(t)\right|_{\varepsilon=0} .
$$

Obviously, $\varphi(a)=0$. Define

$$
f(\varepsilon)=z[x+\varepsilon \eta, \zeta+\varepsilon \Delta \zeta]_{\tau}(b)
$$

Since $z(b)$ is a local extremum, $\dot{f}(0)=0$, and then $\varphi(b)=0$. Using similar arguments to those used in the Proof of Theorem 3.1, we conclude that

$$
\begin{aligned}
\dot{\varphi}(t)= & \left.\frac{d}{d t} \frac{d}{d \varepsilon} z[x+\varepsilon \eta, \zeta+\varepsilon \Delta \zeta]_{\tau}(t)\right|_{\varepsilon=0} \\
= & \left.\frac{d}{d \varepsilon} \frac{d}{d t} z[x+\varepsilon \eta, \zeta+\varepsilon \Delta \zeta]_{\tau}(t)\right|_{\varepsilon=0} \\
= & \partial_{2} L[x, z, \zeta]_{\tau}(t) \cdot \eta(t)+\partial_{3} L[x, z, \zeta]_{\tau} \cdot \eta(t-\tau)+\partial_{4} L[x, z, \zeta]_{\tau}(t) \cdot\left({ }^{C} D_{a^{+}}^{\alpha, \psi} \eta\right)(t) \\
& +\partial_{5} L[x, z, \zeta]_{\tau}(t) \cdot\left({ }^{C} D_{b^{-}}^{\beta, \psi} \eta\right)(t)+\partial_{6} L[x, z, \zeta]_{\tau}(t) \cdot \varphi(t) \\
& +\partial_{7} L[x, z, \zeta]_{\tau}(t) \cdot \eta(a)+\partial_{8} L[x, z, \zeta]_{\tau}(t) \cdot \eta(b)+\partial_{9} L[x, z, \zeta]_{\tau}(t) \cdot \Delta \zeta
\end{aligned}
$$

Observing that $\eta(a)=0$ and solving the last differential equation, we get

$$
\begin{aligned}
& e^{-\int_{a}^{t} \partial_{6} L[x, z, \zeta]_{\tau}(s) d s} \varphi(t)-\varphi(a) \\
& =\int_{a}^{t} e^{-\int_{a}^{s} \partial_{6} L[x, z, \zeta]_{\tau}(\tau) d \tau} \cdot\left(\partial_{2} L[x, z, \zeta](s) \cdot \eta(s)+\partial_{3} L[x, z, \zeta](s) \cdot \eta(s-\tau)\right. \\
& +\partial_{4} L[x, z, \zeta]_{\tau}(s) \cdot\left({ }^{C} D_{a^{+}}^{\alpha, \psi} \eta\right)(s)+\partial_{5} L[x, z, \zeta]_{\tau}(s) \cdot\left({ }^{C} D_{b^{-}}^{\beta, \psi} \eta\right)(s) \\
& \left.+\partial_{8} L[x, z, \zeta]_{\tau}(s) \cdot \eta(b)+\partial_{9} L[x, z, \zeta]_{\tau}(s) \cdot \Delta \zeta\right) d s .
\end{aligned}
$$

Considering $t=b$ and observing that $\varphi(a)=\varphi(b)=0$, we get

$$
\begin{aligned}
& \int_{a}^{b} \lambda(s) \cdot\left(\partial_{2} L[x, z, \zeta](s) \cdot \eta(s)+\partial_{3} L[x, z, \zeta](s) \cdot \eta(s-\tau)\right. \\
& +\partial_{4} L[x, z, \zeta]_{\tau}(s) \cdot\left({ }^{C} D_{a^{+}}^{\alpha, \psi} \eta\right)(s)+\partial_{5} L[x, z, \zeta]_{\tau}(s) \cdot\left({ }^{C} D_{b^{-}}^{\beta, \psi} \eta\right)(s) \\
& \left.+\partial_{8} L[x, z, \zeta]_{\tau}(s) \cdot \eta(b)+\partial_{9} L[x, z, \zeta]_{\tau}(s) \cdot \Delta \zeta\right) d s=0 .
\end{aligned}
$$

Applying the change of variable $s=t+\tau$ and recalling that $\eta$ is null on $[a-\tau, a]$, we obtain that

$$
\int_{a}^{b} \lambda(s) \cdot \partial_{3} L[x, z, \zeta]_{\tau}(s) \cdot \eta(s-\tau) d s=\int_{a}^{b-\tau} \lambda(s+\tau) \cdot \partial_{3} L[x, z, \zeta]_{\tau}(s+\tau) \cdot \eta(s) d s
$$

Observe also that for $t \in[a, b-\tau]$, 


$$
\begin{array}{r}
\left(D_{b^{-}}^{\alpha, \psi} \frac{\lambda \cdot \partial_{4} L[x, z, \zeta]_{\tau}}{\dot{\psi}}\right)(t)=\left(D_{(b-\tau)^{-}}^{\alpha, \psi} \frac{\lambda \cdot \partial_{4} L[x, z, \zeta]_{\tau}}{\dot{\psi}}\right)(t) \\
-\frac{1}{\Gamma(1-\alpha)}\left(\frac{1}{\dot{\psi}(t)} \frac{d}{d t}\right) \int_{b-\tau}^{b} \lambda(s)(\psi(s)-\psi(t))^{-\alpha} \cdot \partial_{4} L[x, z, \zeta]_{\tau}(s) d s
\end{array}
$$

and for $t \in[b-\tau, b]$,

$$
\begin{array}{r}
\left(D_{a^{+}}^{\beta, \psi} \frac{\lambda \cdot \partial_{5} L[x, z, \zeta]_{\tau}}{\dot{\psi}}\right)(t)=\left(D_{(b-\tau)^{+}}^{\beta, \psi} \frac{\lambda \cdot \partial_{5} L[x, z, \zeta]_{\tau}}{\dot{\psi}}\right)(t) \\
+\frac{1}{\Gamma(1-\beta)}\left(\frac{1}{\dot{\psi}(t)} \frac{d}{d t}\right) \int_{a}^{b-\tau} \lambda(s)(\psi(t)-\psi(s))^{-\beta} \cdot \partial_{5} L[x, z, \zeta]_{\tau}(s) d s .
\end{array}
$$

Using fractional integration by parts and (25), we get

$$
\begin{gathered}
\int_{a}^{b} \lambda(t) \partial_{4} L[x, z, \zeta]_{\tau}(t) \cdot\left({ }^{C} D_{a^{+}}^{\alpha, \psi} \eta\right)(t) d t=\int_{a}^{b-\tau}\left(\left(D_{(b-\tau)^{-}}^{\alpha, \psi} \frac{\lambda \cdot \partial_{4} L[x, z, \zeta]_{\tau}}{\dot{\psi}}\right)(t) \cdot \dot{\psi}(t)\right. \\
\left.-\frac{1}{\Gamma(1-\alpha)} \frac{d}{d t} \int_{b-\tau}^{b} \lambda(s)(\psi(s)-\psi(t))^{-\alpha} \cdot \partial_{4} L[x, z, \zeta]_{\tau}(s) d s\right) \cdot \eta(t) d t \\
+\int_{b-\tau}^{b}\left(D_{b^{-}}^{\alpha, \psi} \frac{\lambda \cdot \partial_{4} L[x, z, \zeta]_{\tau}}{\dot{\psi}}\right)(t) \cdot \dot{\psi}(t) \cdot \eta(t) d t+\left[\left(I_{b^{-}}^{1-\alpha, \psi} \frac{\lambda \cdot \partial_{4} L[x, z, \zeta]_{\tau}}{\dot{\psi}}\right)(t) \cdot \eta(t)\right]_{t=a}^{t=b} .
\end{gathered}
$$

Using fractional integration by parts and (26), we get

$$
\begin{aligned}
& \int_{a}^{b} \lambda(t) \cdot \partial_{5} L[x, z, \zeta]_{\tau}(t) \cdot\left({ }^{C} D_{b^{-}}^{\beta, \psi} \eta\right)(t) d t=\int_{a}^{b-\tau}\left(D_{a^{+}}^{\beta, \psi} \frac{\lambda \cdot \partial_{5} L[x, z, \zeta]_{\tau}}{\dot{\psi}}\right)(t) \cdot \dot{\psi}(t) \cdot \eta(t) d t \\
&+ \int_{b-\tau}^{b}\left(\left(D_{(b-\tau)^{+}}^{\beta, \psi} \frac{\lambda \cdot \partial_{5} L[x, z, \zeta]_{\tau}}{\dot{\psi}}\right)(t) \cdot \dot{\psi}(t)\right. \\
&\left.+\frac{1}{\Gamma(1-\beta)} \frac{d}{d t} \int_{a}^{b-\tau} \lambda(s)(\psi(t)-\psi(s))^{-\beta} \cdot \partial_{5} L[x, z, \zeta]_{\tau}(s) d s\right) \cdot \eta(t) d t \\
&-\left[\left(I_{a^{+}}^{1-\beta, \psi} \frac{\lambda \cdot \partial_{5} L[x, z, \zeta]_{\tau}}{\dot{\psi}}\right)(t) \cdot \eta(t)\right]_{t=a}^{t=b} .
\end{aligned}
$$

Introducing (24), (27), and (28) into Eq. (23), we can conclude that

$$
\begin{array}{r}
\int_{a}^{b-\tau}\left(\lambda(t) \cdot \partial_{2} L[x, z, \zeta]_{\tau}(t)+\lambda(t+\tau) \cdot \partial_{3} L[x, z, \zeta]_{\tau}(t+\tau)+\left(D_{(b-\tau)^{-}}^{\alpha, \psi} \frac{\lambda \cdot \partial_{4} L[x, z, \zeta]_{\tau}}{\dot{\psi}}\right)(t) \dot{\psi}(t)\right. \\
\left.-\frac{1}{\Gamma(1-\alpha)} \frac{d}{d t} \int_{b-\tau}^{b} \lambda(s)(\psi(s)-\psi(t))^{-\alpha} \cdot \partial_{4} L[x, z, \zeta]_{\tau} d s+\left(D_{a^{+}}^{\beta, \psi} \frac{\lambda(t) \cdot \partial_{5} L[x, z, \zeta]_{\tau}}{\dot{\psi}}\right)(t) \dot{\psi}(t)\right) \cdot \eta(t) d t \\
+\int_{b-\tau}^{b}\left(\lambda(t) \cdot \partial_{2} L[x, z, \zeta]_{\tau}(t)+\left(D_{b^{-}}^{\alpha, \psi} \frac{\lambda \cdot \partial_{4} L[x, z, \zeta]_{\tau}}{\dot{\psi}}\right)(t) \dot{\psi}(t)+\left(D_{(b-\tau)^{+}}^{\beta, \psi} \frac{\lambda \cdot \partial_{5} L[x, z, \zeta]_{\tau}}{\dot{\psi}}\right)(t) \dot{\psi}(t)\right. \\
\left.+\frac{1}{\Gamma(1-\beta)} \frac{d}{d t} \int_{a}^{b-\tau} \lambda(s) \cdot(\psi(t)-\psi(s))^{-\beta} \cdot \partial_{5} L[x, z, \zeta]_{\tau}(s) d s\right) \cdot \eta(t) d t \\
+\left[\left(I_{b^{-}}^{1-\alpha, \psi} \frac{\lambda \cdot \partial_{4} L[x, z, \zeta]_{\tau}}{\dot{\psi}}\right)(t) \cdot \eta(t)\right]_{t=a}^{t=b}-\left[\left(I_{a^{+}}^{1-\beta, \psi} \frac{\lambda \cdot \partial_{5} L[x, z, \zeta]_{\tau}}{\dot{\psi}}\right)(t) \cdot \eta(t)\right]_{t=a}^{t=b} \\
+\int_{a}^{b} \lambda(t)\left(\partial_{8} L[x, z, \zeta]_{\tau}(t) \cdot \eta(b)+\partial_{9} L[x, z, \zeta]_{\tau}(t) \cdot \Delta \zeta\right) d t=0 .
\end{array}
$$

With appropriate choices for $\eta$ and $\Delta \zeta$, we get the Euler-Lagrange equations [(18) and (19)], the necessary condition (20), and the natural boundary condition (21). 
Remark 4. We remark that

1. if the delay is removed, that is, if we take $\tau=0$, problem $\left(\mathcal{P}_{2}\right)$ reduces to problem $\left(\mathcal{P}_{1}\right)$. Therefore, Theorem 3.1 is a corollary of Theorem 3.2;

2. since the state value $x(a)$ is fixed, the Lagrangian's explicit dependence on $x(a)$ is irrelevant; we choose to include $x(a)$ in $L$ to maintain coherence with the other variational problems studied in this paper;

3. if $\psi$ is the identity and $\alpha \rightarrow 1^{-}$and $\beta \rightarrow 1^{-}$, then we get the classical non-standard Herglotz variational principle with time delay that, to the best of authors's knowlodge, is new in the literature.

\section{Herglotz fractional variational principle with higher-order derivatives}

In what follows, we will use the following notation:

$$
\begin{aligned}
{[x, z, \zeta]_{n}(t) } & :=\left(t, x(t),\left({ }^{C} D_{a^{+}}^{\alpha_{1}, \psi} x\right)(t),\left({ }^{C} D_{b^{-}}^{\beta_{1}, \psi} x\right)(t), \ldots,\left({ }^{C} D_{a^{+}}^{\alpha_{n}, \psi} x\right)(t),\left({ }^{C} D_{b^{-}}^{\beta_{n}, \psi} x\right)(t), z(t), x(a), x(b), \zeta\right), \\
{[x, \zeta]_{n}(t) } & :=\left(t, x(t),\left({ }^{C} D_{a^{+}}^{\alpha_{1}, \psi} x\right)(t),\left({ }^{C} D_{b^{-}}^{\beta_{1}, \psi} x\right)(t), \ldots,\left({ }^{C} D_{a^{+}}^{\alpha_{n}, \psi} x\right)(t),\left({ }^{C} D_{b^{-}}^{\beta_{n}, \psi} x\right)(t), x(a), x(b), \zeta\right),
\end{aligned}
$$

where $n$ is a positive integer and $\left.\alpha_{i}, \beta_{i} \in\right] i-1, i[$, for all $i \in\{1, \ldots, n\}$. The problem that we deal in this subsection is stated next.

Problem $\left(\mathcal{P}_{3}\right)$ : Determine $x \in C^{n}([a, b], \mathbb{R}), z \in C^{1}([a, b], \mathbb{R})$, and $\zeta \in \mathbb{R}$ that extremize

$$
z(b)
$$

where $(x, z, \zeta)$ satisfies the differential equation

$$
\dot{z}(t)=L[x, z, \zeta]_{n}(t), \quad t \in[a, b]
$$

subject to the boundary conditions

$$
z(a)=\gamma \quad \text { and } \quad x^{(i)}(a)=x_{a}^{i}, x^{(i)}(b)=x_{b}^{i}, i \in\{1, \ldots, n-1\}
$$

for fixed $\gamma, x_{a}^{i}, x_{b}^{i} \in \mathbb{R}$. We assume that for all $i,{ }^{C} D_{a^{+}}^{\alpha_{i}, \psi} x,{ }^{C} D_{b^{-}}^{\beta_{i}, \psi} x \in C^{1}([a, b], \mathbb{R}), L:[a, b] \times \mathbb{R}^{2 n+5} \rightarrow \mathbb{R}$ is of class $C^{1}$, and exist and are continuous the maps

$$
t \mapsto\left(D_{b^{-}}^{\alpha_{i}, \psi} \frac{\lambda \cdot \partial_{2 i+1} L[x, z, \zeta]_{n}}{\dot{\psi}}\right)(t) \quad \text { and } \quad t \mapsto\left(D_{a^{+}}^{\beta_{i}, \psi} \frac{\lambda \cdot \partial_{2 i+2} L[x, z, \zeta]_{n}}{\dot{\psi}}\right)(t), t \in[a, b]
$$

for all admissible $(x, z, \zeta)$, where

$$
\lambda(t):=e^{-\int_{a}^{t} \partial_{2 n+3} L[x, z, \zeta]_{n}(s) d s}, \quad t \in[a, b] .
$$

Theorem 3.3 (Non-standard Herglotz's fractional variational principle with higher-order derivatives). If ( $x$, $z$, $\zeta$ ) is a solution of problem $\left(\mathcal{P}_{3}\right)$, then $(x, z, \zeta)$ satisfies the fractional Euler-Lagrange equation

$$
\begin{aligned}
\lambda(t) \cdot \partial_{2} L[x, z, \zeta]_{n}(t) & +\sum_{i=1}^{n}\left[\left(D_{b^{-}}^{\alpha_{i}, \psi} \frac{\lambda \cdot \partial_{2 i+1} L[x, z, \zeta]_{n}}{\dot{\psi}}\right)(t) \cdot \dot{\psi}(t)\right. \\
& \left.+\left(D_{a^{+}}^{\beta_{i}, \psi} \frac{\lambda \cdot \partial_{2 i+2} L[x, z, \zeta]_{n}}{\dot{\psi}}\right)(t) \cdot \dot{\psi}(t)\right]=0,
\end{aligned}
$$

for all $t \in[a, b]$ and

$$
\int_{a}^{b} \lambda(t) \cdot \partial_{2 n+6} L[x, z, \zeta]_{n}(t) d t=0
$$

In addition,

1. if $x(a)$ is free, then $(x, z, \zeta)$ satisfies the following condition: 


$$
\begin{aligned}
\int_{a}^{b} \lambda(t) \cdot \partial_{2 n+4} L[x, z, \zeta]_{n}(t) d t=\sum_{i=1}^{n} & {\left[\left(-\frac{1}{\dot{\psi}} \frac{d}{d t}\right)^{i-1}\left(I_{b^{-}}^{i-\alpha_{i}, \psi} \frac{\lambda \cdot \partial_{2 i+1} L[x, z, \zeta]_{n}}{\dot{\psi}}\right)(a)\right.} \\
& \left.-\left(\frac{1}{\dot{\psi}} \frac{d}{d t}\right)^{i-1}\left(I_{a^{+}}^{i-\beta_{i} \psi} \frac{\lambda \cdot \partial_{2 i+2} L[x, z, \zeta]_{n}}{\dot{\psi}}\right)(a)\right],
\end{aligned}
$$

2. if $x(b)$ is free, then $(x, z, \zeta)$ satisfies the following condition:

$$
\begin{aligned}
\int_{a}^{b} \lambda(t) \cdot \partial_{2 n+5} L[x, z, \zeta]_{n}(t) d t & =\sum_{i=1}^{n}\left[\left(\frac{1}{\dot{\psi}} \frac{d}{d t}\right)^{i-1}\left(I_{a^{+}}^{i-\beta_{i} \psi} \frac{\lambda \cdot \partial_{2 i+2} L[x, z, \zeta]_{n}}{\dot{\psi}}\right)(b)\right. \\
& \left.-\left(-\frac{1}{\dot{\psi}} \frac{d}{d t}\right)^{i-1}\left(I_{b^{-}}^{i-\alpha_{i},} \frac{\lambda \cdot \partial_{2 i+1} L[x, z, \zeta]_{n}}{\dot{\psi}}\right)(b)\right] .
\end{aligned}
$$

Proof. Let $\eta \in C^{n}([a, b], \mathbb{R})$ be an arbitrary function satisfying the conditions $\eta^{(i)}(a)=0=\eta^{(i)}(b), i \in\{1, \ldots, n-1\}$, and its Caputo fractional derivatives are continuously differentiable, and $\Delta \zeta$ be an arbitrary real number. Defining $\varphi:[a, b] \rightarrow \mathbb{R}$ as

$$
\varphi(t)=\left.\frac{d}{d \varepsilon} z[x+\varepsilon \eta, \zeta+\varepsilon \Delta \zeta]_{n}(t)\right|_{\varepsilon=0},
$$

$\varphi(a)=0=\varphi(b)$ and

$$
\begin{aligned}
\dot{\varphi}(t)= & \partial_{2} L[x, z, \zeta]_{n}(t) \cdot \eta(t)+\sum_{i=1}^{n}\left[\partial_{2 i+1} L[x, z, \zeta]_{n}(t) \cdot\left({ }^{C} D_{a^{+}}^{\alpha_{i} \psi} \eta\right)(t)+\right. \\
& \left.\partial_{2 i+2} L[x, z, \zeta]_{n}(t) \cdot\left({ }^{C} D_{b^{-}}^{\beta_{i} \psi} \eta\right)(t)\right]+\partial_{2 n+3} L[x, z, \zeta]_{n}(t) \cdot \varphi(t)+ \\
& \partial_{2 n+4} L[x, z, \zeta]_{n}(t) \cdot \eta(a)+\partial_{2 n+5} L[x, z, \zeta]_{n}(t) \cdot \eta(b)+\partial_{2 n+6} L[x, z, \zeta]_{n}(t) \cdot \Delta \zeta .
\end{aligned}
$$

The solution of the last equation is the function $\varphi$ defined by

$$
\begin{array}{r}
\lambda(t) \varphi(t)-\varphi(a)=\int_{a}^{t} \lambda(s) \cdot\left(\partial_{2} L[x, z, \zeta]_{n}(s) \cdot \eta(s)+\sum_{i=1}^{n}\left[\partial_{2 i+1} L[x, z, \zeta]_{n}(s) \cdot\left({ }^{C} D_{a^{+}}^{\alpha_{i} \psi} \eta\right)(s)\right.\right. \\
\left.+\partial_{2 i+2} L[x, z, \zeta]_{n}(s) \cdot\left({ }^{C} D_{b^{-}}^{\beta_{i} \psi} \eta\right)(s)\right]+\partial_{2 n+4} L[x, z, \zeta]_{n}(s) \cdot \eta(a) \\
\left.+\partial_{2 n+5} L[x, z, \zeta]_{n}(s) \cdot \eta(b)+\partial_{2 n+6} L[x, z, \zeta]_{n}(s) \cdot \Delta \zeta\right) d s .
\end{array}
$$

Evaluated at $t=b$ and using fractional integration by parts, we obtain

$$
\begin{aligned}
& \int_{a}^{b}\left(\lambda(s) \cdot \partial_{2} L[x, z, \zeta]_{n}(s)+\sum_{i=1}^{n}\left[\left(D_{b^{-}}^{\alpha_{i} \psi} \frac{\lambda \cdot \partial_{2 i+1} L[x, z, \zeta]_{n}}{\dot{\psi}}\right)(s) \cdot \dot{\psi}(s)\right.\right. \\
& \left.\left.+\left(D_{a^{+}}^{\beta_{i},} \frac{\lambda \cdot \partial_{2 i+2} L[x, z, \zeta]_{n}}{\dot{\psi}}\right)(s) \cdot \dot{\psi}(s)\right]\right) \eta(s) d s \\
& +\sum_{i=1}^{n}\left[\left(-\frac{1}{\dot{\psi}(s)} \frac{d}{d s}\right)^{i-1}\left(I_{b^{-}}^{i-\alpha_{i} \psi} \frac{\lambda \cdot \partial_{2 i+1} L[x, z, \zeta]_{n}}{\dot{\psi}}\right)(s) \cdot \eta(s)\right]_{s=a}^{s=b} \\
& -\sum_{i=1}^{n}\left[\left(\frac{1}{\dot{\psi}(s)} \frac{d}{d s}\right)^{i-1}\left(I_{a^{+}}^{i-\beta_{i} \psi} \frac{\lambda \cdot \partial_{2 i+2} L[x, z, \zeta]_{n}}{\dot{\psi}}\right)(s) \cdot \eta(s)\right]_{s=a}^{s=b} \\
& +\int_{a}^{b} \lambda(s) \cdot\left(\partial_{2 n+4} L[x, z, \zeta]_{n}(s) \cdot \eta(a)+\partial_{2 n+5} L[x, z, \zeta]_{n}(s) \cdot \eta(b)\right. \\
& \left.+\partial_{2 n+6} L[x, z, \zeta]_{n}(s) \cdot \Delta \zeta\right) d s=0 .
\end{aligned}
$$

From the arbitrariness of $\eta$ and $\Delta \zeta$, we deduce the necessary conditions [(30)-(33)].

We remark that when $L$ does not depends on $z$, we obtain (Ref. 10, Theorem 3.3). 


\section{Herglotz fractional variational principle with several independent variables}

In this final subsection, we consider the case where the state function depends on several independent variables. Let $\Omega=\prod_{i=1}^{n}\left[a_{i}, b_{i}\right]$ and $P=[a, b] \times \Omega$. The time variable is denoted by $t \in[a, b]$, and the spatial coordinates are denoted by $s=\left(s_{1}, \ldots, s_{n}\right) \in \Omega$. The following notations are also used:

$$
\begin{gathered}
{[x, z, \zeta](t, s):=\left(t, s, x(t, s),\left({ }^{C} D_{+}^{\alpha, \psi} x\right)(t, s),\left({ }^{C} D_{-}^{\beta, \psi} x\right)(t, s), z(t), x(a, s), x(b, s), \zeta\right)} \\
{[x, \zeta](t, s):=\left(t, s, x(t, s),\left({ }^{C} D_{+}^{\alpha, \psi} x\right)(t, s),\left({ }^{C} D_{-}^{\beta, \psi} x\right)(t, s), x(a, s), x(b, s), \zeta\right) .}
\end{gathered}
$$

Here,

$$
\left({ }^{C} D_{+}^{\alpha, \psi} x\right)(t, s):=\left(\left({ }^{C} D_{a^{+}}^{\alpha, \psi} x\right)(t, s),\left({ }^{C} D_{a_{1}^{+}}^{\alpha, \psi} x\right)(t, s), \ldots,\left({ }^{C} D_{a_{n}^{+}}^{\alpha, \psi} x\right)(t, s)\right) \in \mathbb{R}^{n+1}
$$

and

$$
\left({ }^{C} D_{-}^{\beta, \psi} x\right)(t, s):=\left(\left({ }^{C} D_{b^{-}}^{\beta, \psi} x\right)(t, s),\left({ }^{C} D_{b_{1}^{-}}^{\beta, \psi} x\right)(t, s), \ldots,\left({ }^{C} D_{b_{n}^{-}}^{\beta, \psi} x\right)(t, s)\right) \in \mathbb{R}^{n+1}
$$

where ${ }^{C} D_{a^{+}}^{\alpha, \psi} x$ and ${ }^{C} D_{b^{-}}^{\beta, \psi} x$ denote the partial left and right sided fractional derivatives of $x$, with respect to the variable $t$, and for $i \in\{1, \ldots, n\}$, ${ }^{C} D_{a_{i}^{+}}^{\alpha, \psi} x$ and ${ }^{C} D_{b_{i}^{-}}^{\beta, \psi} x$ denote the partial left and right sided fractional derivatives of $x$, with respect to the variable $s_{i}$. It is also assumed that the domain of function $\psi$ contains the intervals $[a, b]$ and $\left[a_{i}, b_{i}\right]$, for $i \in\{1, \ldots, n\}$.

Problem $\left(\mathcal{P}_{4}\right)$ : Determine $x \in C^{1}(P, \mathbb{R}), z \in C^{1}([a, b], \mathbb{R})$, and $\zeta \in \mathbb{R}$ that extremize

$$
z(b)
$$

where $(x, z, \zeta)$ satisfies the differential equation

$$
\dot{z}(t)=\int_{\Omega} L[x, z, \zeta](t, s) d^{n} s, \quad t \in[a, b],
$$

and

$$
z(a)=\gamma
$$

for a given $\gamma \in \mathbb{R}, d^{n} s=d s_{1} \ldots d s_{n}$. It is assumed that ${ }^{C} D_{a^{+}}^{\alpha, \psi} x,{ }^{C} D_{b^{-}}^{\beta, \psi} x,{ }^{C} D_{a_{i}^{+}}^{\alpha, \psi} x$, and ${ }^{C} D_{b_{i}^{-}}^{\beta, \psi} x$ are all continuously differentiable functions, for $i \in\{1, \ldots, n\}$, and

1. $\quad L:[a, b] \times \mathbb{R}^{3 n+7} \rightarrow \mathbb{R}$ is of class $C^{1}$;

2. functions

$$
\begin{gathered}
(t, s) \mapsto\left(D_{b^{-}}^{\alpha, \psi} \frac{\lambda \cdot \partial_{n+3} L[x, z, \zeta]}{\dot{\psi}}\right)(t, s), \quad(t, s) \mapsto\left(D_{a^{+}}^{\beta, \psi} \frac{\lambda \cdot \partial_{2 n+4} L[x, z, \zeta]}{\dot{\psi}}\right)(t, s), \\
(t, s) \mapsto\left(D_{b_{i}^{-}}^{\alpha, \psi} \frac{\lambda \cdot \partial_{n+3+i} L[x, z, \zeta]}{\dot{\psi}}\right)(t, s), \quad(t, s) \mapsto\left(D_{a_{i}^{+}}^{\beta, \psi} \frac{\lambda \cdot \partial_{2 n+4+i} L[x, z, \zeta]}{\dot{\psi}}\right)(t, s),
\end{gathered}
$$

exist and are continuous on $P$, for all admissible triplet $(x, z, \zeta)$ and for all $i \in\{1, \ldots, n\}$, where

$$
\lambda(t):=e^{-\int_{a}^{t} \int_{\Omega} \partial_{3 n+5} L[x, z, \zeta](\tau, s) d^{n} s d \tau}, \quad t \in[a, b]
$$

Theorem 3.4 (Non-standard Herglotz's fractional variational principle with several independent variables). If ( $x, z, \zeta)$ is a solution of problem $\left(\mathcal{P}_{4}\right)$, then $(x, z, \zeta)$ satisfies the fractional Euler-Lagrange equation

$$
\begin{array}{r}
\lambda(t) \cdot \partial_{n+2} L[x, z, \zeta](t, s)+\left(D_{b^{-}}^{\alpha, \psi} \frac{\lambda \cdot \partial_{n+3} L[x, z, \zeta]}{\dot{\psi}}\right)(t, s) \cdot \dot{\psi}(t) \\
+\left(D_{a^{+}}^{\beta, \psi} \frac{\lambda \cdot \partial_{2 n+4} L[x, z, \zeta]}{\dot{\psi}}\right)(t, s) \cdot \dot{\psi}(t)+\sum_{i=1}^{n}\left[\left(D_{b_{i}^{-}}^{\alpha, \psi} \frac{\lambda \cdot \partial_{n+3+i} L[x, z, \zeta]}{\dot{\psi}}\right)(t, s) \cdot \dot{\psi}(t)\right. \\
\left.+\left(D_{a_{i}^{+}}^{\beta, \psi} \frac{\lambda \cdot \partial_{2 n+4+i} L[x, z, \zeta]}{\dot{\psi}}\right)(t, s) \cdot \dot{\psi}(t)\right]=0,
\end{array}
$$


for all $(t, s) \in P$ and

$$
\int_{a}^{b} \int_{\Omega} \lambda(t) \cdot \partial_{3 n+8} L[x, z, \zeta](t, s) d^{n} s d t=0
$$

In addition,

1. if $x(a, \cdot)$ is free, then

$$
\begin{aligned}
\int_{a}^{b} \int_{\Omega} \lambda(t) \cdot \partial_{3 n+6} L[x, z, \zeta](t, s) d t & =\int_{\Omega}\left(I_{b^{-}}^{1-\alpha, \psi} \frac{\lambda \cdot \partial_{n+3} L[x, z, \zeta]}{\dot{\psi}}\right)(a, s) d^{n} s \\
& -\int_{\Omega}\left(I_{a^{+}}^{1-\beta, \psi} \frac{\lambda \cdot \partial_{2 n+4} L[x, z, \zeta]}{\dot{\psi}}\right)(a, s) d^{n} s
\end{aligned}
$$

2. if $x(b, \cdot)$ is free, then

$$
\begin{aligned}
\int_{a}^{b} \int_{\Omega} \lambda(t) \cdot \partial_{3 n+7} L[x, z, \zeta](t, s) d t & =\int_{\Omega}\left(I_{a^{+}}^{1-\beta, \psi} \frac{\lambda \cdot \partial_{2 n+4} L[x, z, \zeta]}{\dot{\psi}}\right)(b, s) d^{n} s \\
& -\int_{\Omega}\left(I_{b^{-}}^{1-\alpha, \psi} \frac{\lambda \cdot \partial_{n+3} L[x, z, \zeta]}{\dot{\psi}}\right)(b, s) d^{n} s
\end{aligned}
$$

Proof. Let $\eta \in C^{1}(P, \mathbb{R})$ such that its Caputo fractional derivatives are continuously differentiable and $\Delta \zeta$ be an arbitrary real number. For simplicity, we will assume that for any $i \in\{1, \ldots, n\}$, if $s_{i}=a_{i}$ or $s_{i}=b_{i}$, then $\eta(t, s)=0, t \in[a, b]$. If we define

$$
\varphi(t)=\left.\frac{d}{d \varepsilon} z[x+\varepsilon \eta, \zeta+\varepsilon \Delta \zeta](t)\right|_{\varepsilon=0}, \quad t \in[a, b],
$$

then $\varphi(a)=0=\varphi(b)$. In addition, since

$$
\begin{aligned}
\dot{\varphi}(t)= & \int_{\Omega}\left[\partial_{n+2} L[x, z, \zeta](t, s) \cdot \eta(t, s)+\partial_{n+3} L[x, z, \zeta](t, s) \cdot\left({ }^{C} D_{a^{+}}^{\alpha, \psi} \eta\right)(t, s)\right. \\
& +\partial_{2 n+4} L[x, z, \zeta](t, s) \cdot\left({ }^{C} D_{b^{-}}^{\beta, \psi} \eta\right)(t, s) \\
& +\sum_{i=1}^{n}\left[\partial_{n+3+i} L[x, z, \zeta](t, s) \cdot\left({ }^{C} D_{a_{i}^{+}}^{\alpha, \psi} \eta\right)(t, s)+\partial_{2 n+4+i} L[x, z, \zeta](t, s) \cdot\left({ }^{C} D_{b_{i}^{-}}^{\beta, \psi} \eta\right)(t, s)\right] \\
& +\partial_{3 n+5} L[x, z, \zeta](t) \cdot \varphi(t)+\partial_{3 n+6} L[x, z, \zeta](t, s) \cdot \eta(a, s) \\
& \left.+\partial_{3 n+7} L[x, z, \zeta](t, s) \cdot \eta(b, s)+\partial_{3 n+8} L[x, z, \zeta](t, s) \cdot \Delta \zeta\right] d^{n} s,
\end{aligned}
$$

solving the differential equation and using the fact that $\varphi(a)=0=\varphi(b)$, we get that

$$
\begin{gathered}
\int_{a}^{b} \int_{\Omega} \lambda(t) \cdot\left(\partial_{n+2} L[x, z, \zeta](t, s) \cdot \eta(t, s)\right. \\
+\partial_{n+3} L[x, z, \zeta](t, s) \cdot\left({ }^{C} D_{a^{+}}^{\alpha, \psi} \eta\right)(t, s)+\partial_{2 n+4} L[x, z, \zeta](t, s) \cdot\left({ }^{C} D_{b^{-}}^{\beta, \psi} \eta\right)(t, s) \\
+\sum_{i=1}^{n}\left[\partial_{n+3+i} L[x, z, \zeta](t, s) \cdot\left({ }^{C} D_{a_{i}^{+}}^{\alpha, \psi} \eta\right)(t, s)+\partial_{2 n+4+i} L[x, z, \zeta](t, s) \cdot\left({ }^{C} D_{b_{i}^{-}}^{\beta, \psi}\right)(t, s)\right] \\
\left.+\partial_{3 n+6} L[x, z, \zeta](t, s) \cdot \eta(a, s)+\partial_{3 n+7} L[x, z, \zeta](t, s) \cdot \eta(b, s)+\partial_{3 n+8} L[x, z, \zeta](t, s) \cdot \Delta \zeta\right) d^{n} s d t=0 .
\end{gathered}
$$

Using fractional integration by parts, we get 


$$
\begin{gathered}
\int_{a}^{b} \int_{\Omega}\left(\lambda(t) \cdot \partial_{n+2} L[x, z, \zeta](t, s)+\left(D_{b^{-}}^{\alpha, \psi} \frac{\lambda \cdot \partial_{n+3} L[x, z, \zeta]}{\dot{\psi}}\right)(t, s) \cdot \dot{\psi}(t)\right. \\
+\left(D_{a^{+}}^{\beta, \psi} \frac{\lambda \cdot \partial_{2 n+4} L[x, z, \zeta]}{\dot{\psi}}\right)(t, s) \cdot \dot{\psi}(t)+\sum_{i=1}^{n}\left[\left(D_{b_{i}^{-}}^{\alpha, \psi} \frac{\lambda \cdot \partial_{n+3+i} L[x, z, \zeta]}{\dot{\psi}}\right)(t, s) \cdot \dot{\psi}(t)\right. \\
\left.+\left(D_{a_{i}^{+}}^{\beta, \psi} \frac{\lambda \cdot \partial_{2 n+4+i} L[x, z, \zeta]}{\dot{\psi}}\right)(t, s) \cdot \dot{\psi}(t)\right] \cdot \eta(t, s) d^{n} s d t \\
+\left[\int_{\Omega}\left(I_{b^{-}}^{1-\alpha, \psi} \frac{\lambda \cdot \partial_{n+3} L[x, z, \zeta]}{\dot{\psi}}\right)(t, s) \cdot \eta(t, s) d^{n} s\right]_{t=a}^{t=b} \\
-\left[\int_{\Omega}\left(I_{a^{+}}^{1-\beta, \psi} \frac{\lambda \cdot \partial_{2 n+4} L[x, z, \zeta]}{\dot{\psi}}\right)(t, s) \cdot \eta(t, s) d^{n} s\right]_{t=a}^{t=b} \\
+\int_{a}^{b} \int_{\Omega} \lambda(t)\left(\partial_{3 n+6} L[x, z, \zeta](t, s) \cdot \eta(a, s)+\partial_{3 n+7} L[x, z, \zeta](t, s) \cdot \eta(b, s)\right. \\
\left.+\partial_{3 n+8} L[x, z, \zeta](t, s) \cdot \Delta \zeta\right) d^{n} s d t=0 .
\end{gathered}
$$

From the arbitrariness of $\eta$ and $\Delta \zeta$, we obtain Eqs. (36)-(39).

\section{ILLUSTRATIVE EXAMPLES}

In order to illustrate our results, we present two examples that cannot be solved using the available results in the literature.

Example 1. Consider the following differential equation, involving the left sided fractional derivative,

$$
\left\{\begin{array}{l}
\dot{z}(t)=\left[\left({ }^{C} D_{0^{+}}^{\alpha, \psi} x\right)(t)-\frac{6(\psi(t)-\psi(0))^{3-\alpha}}{\Gamma(4-\alpha)}\right]^{3}+\cos ^{2}(z(t))+x^{2}(0)+(\zeta-1)^{2}, t \in[0,1], \\
z(0)=0, \quad x(1)=(\psi(1)-\psi(0))^{3},
\end{array}\right.
$$

where $\alpha \in] 0,1[$ and $x(0)$ is free. The necessary conditions of optimality for this problem, given by Theorem 3.1, are as follows:

1. $D_{1^{-}}^{\alpha, \psi}\left(\frac{3 \lambda(t)\left[\left({ }^{C} D_{0^{+}}^{\alpha, \psi} x\right)(t)-\frac{6(\psi(t)-\psi(0))^{3-\alpha}}{\Gamma(4-\alpha)}\right]^{2}}{\dot{\psi}}\right)(t) \cdot \dot{\psi}(t)=0$,

2. $\int_{0}^{1} 2 \lambda(t)(\zeta-1) d t=0$,

3. $\int_{0}^{1} 2 \lambda(t) x(0) d t=I_{1^{-}}^{1-\alpha, \psi}\left(\frac{3 \lambda(t)\left[\left({ }^{C} D_{0^{+}}^{\alpha, \psi} x\right)(t)-\frac{6(\psi(t)-\psi(0))^{3-\alpha}}{\Gamma(4-\alpha)}\right]^{2}}{\dot{\psi}}\right)(0)$,

where

$$
\lambda(t)=e^{\int_{0}^{t} 2 \sin (z(s)) \cos (z(s)) d s}
$$

It is easy to check that functions $x(t)=(\psi(t)-\psi(0))^{3}$ and $z(t)=\arctan (t)$, together with the parameter $\zeta=1$, satisfy the necessary optimality conditions since

$$
\left({ }^{C} D_{0^{+}}^{\alpha, \psi}(\psi(\cdot)-\psi(0))^{3}\right)(t)=\frac{6(\psi(t)-\psi(0))^{3-\alpha}}{\Gamma(4-\alpha)} .
$$

Example 2. For this second example, we consider $\alpha \epsilon] 1,2[$ and $\psi(t)=t$. The system is given by

$$
\left\{\begin{array}{l}
\dot{z}(t)=-\frac{e^{t} 5 ! t^{2-\alpha}}{2 \Gamma(6-\alpha)} x^{2}(t)+\left({ }^{C} D_{1^{-}}^{\alpha, \psi} x\right)(t) e^{t} t^{5}+z(t)+(x(1)-K)^{2}+\zeta^{2}, t \in[0,1], \\
z(0)=\gamma, \quad x(0)=0,
\end{array}\right.
$$

with $K=1-5 ! /\left(2\left(1-e^{-1}\right) \Gamma(7-\alpha)\right), \gamma \in \mathbb{R}$, and $x(1)$ is free. In this case, $\lambda(t)=e^{-t}$. By Theorem 3.3, a solution of this Herglotz type problem must satisfy Eq. (30),

$$
-x(t) \frac{5 !}{\Gamma(6-\alpha)} t^{2-\alpha}+\frac{5 !}{\Gamma(6-\alpha)} t^{5-\alpha}=0, \quad t \in[0,1]
$$


which is verified for $x(t)=t^{3}$. Equation (31) holds if and only if $\zeta=0$. Finally, Eq. (33) reads as

$$
\int_{0}^{1} 2 e^{-t}(x(1)-K) d t=\frac{d}{d t}\left(I_{0^{+}}^{2-\alpha, \psi} t^{5}\right)(1) .
$$

Simple calculations lead to

$$
\frac{d}{d t}\left(I_{0^{+}}^{2-\alpha, \psi} t^{5}\right)=\frac{5 !}{\Gamma(7-\alpha)} t^{6-\alpha},
$$

and so $x(t)=t^{3}$ also verifies Eq. (41). Function $z$ can be found by replacing $x(t)=t^{3}$ and $\zeta=0$ in system (40) and then solving it.

\section{ACKNOWLEDGMENTS}

This work was supported by Portuguese funds through the CIDMA-Center for Research and Development in Mathematics and Applications, and the Portuguese Foundation for Science and Technology (FCT-Fundação para a Ciência e a Tecnologia), within project Grant No. UIDB/04106/2020.

\section{DATA AVAILABILITY}

Data sharing is not applicable to this article as no new data were created or analyzed in this study.

\section{REFERENCES}

${ }^{1}$ L. Abrunheiro, L. Machado, and N. Martins, “The Herglotz variational problem on spheres and its optimal control approach,” J. Math. Anal. 7(1), 12-22 (2016).

${ }^{2}$ O. P. Agrawal, "Fractional variational calculus and the transversality conditions," J. Phys. A 39(33), 10375-10384 (2006).

${ }^{3}$ R. Almeida and A. B. Malinowska, "Fractional variational principle of Herglotz," Discrete Contin. Dyn. Syst., Ser. B 19(8), 2367-2381 (2014).

${ }^{4}$ R. Almeida, "Fractional variational problems depending on indefinite integrals and with delay," Bull. Malays. Math. Sci. Soc. 39, 1515-1528 (2016).

${ }^{5}$ R. Almeida, "A Caputo fractional derivative of a function with respect to another function," Commun. Nonlinear Sci. Numer. Simul. 44, 460-481 (2017).

${ }^{6}$ R. Almeida, "Optimality conditions for fractional variational problems with free terminal time," Discrete Contin. Dyn. Syst., Ser. S 11(1), 1-19 (2018).

${ }^{7}$ R. Almeida, "Further properties of Osler's generalized fractional integrals and derivatives with respect to another function," Rocky Mt. J. Math. 49(8), 2459-2493 (2019).

${ }^{8}$ R. Almeida, S. Pooseh, and D. F. M. Torres, Computational Methods in the Fractional Calculus of Variations (Imperial College Press, London, 2015).

${ }^{9} \mathrm{R}$. Almeida and N. Martins, "A generalization of a fractional variational problem with dependence on the boundaries and a real parameter" (submitted).

${ }^{10} \mathrm{R}$. Almeida and N. Martins, "New variational problems with an action depending on generalized fractional derivatives, the free endpoints conditions and a real parameter" (submitted).

${ }^{11}$ B. van Brunt, The Calculus of Variations (Universitext) (Springer-Verlag, New York, 2004).

${ }^{12}$ C.-Q. Fang, H.-Y. Sun, and J.-P. Gu, “Application of fractional calculus methods to viscoelastic response of amorphous shape memory polymers," J. Mech. 31(4), 427-432 (2015).

${ }^{13}$ B. Georgieva and R. Guenther, "First Noether-type theorem for the generalized variational principle of Herglotz," Topol. Methods Nonlinear Anal. 2(20), 261-273 (2002).

${ }^{14}$ B. Georgieva and R. Guenther, "Second Noether-type theorem for the generalized variational principle of Herglotz," Topol. Methods Nonlinear Anal. 26(2), 307-314 (2005).

${ }^{15}$ R. B. Guenther, J. A. Gottsch, and D. B. Kramer, “The Herglotz algorithm for constructing canonical transformations,” SIAM Rev. 38(2), 287-293 (1996).

${ }^{16}$ R. B. Guenther and J. A. Gottsch, "The Herglotz lectures on contact transformations and Hamiltonian systems," in Lecture Notes in Nonlinear Analysis (Juliusz Schauder Center for Nonlinear Studies, Nicholas Copernicus University, Torún, 1996), Vol. 1.

${ }^{17}$ G. Herglotz, Berührungstransformationen, Lectures at the University of Göttingen (University of Göttingen, Göttingen, 1930).

${ }^{18} \mathrm{~K}$. A. Hoffman, "Stability results for constrained calculus of variations problems: An analysis of the twisted elastic loop," Proc. R. Soc. A 461, 1357-1381 (2005).

${ }^{19}$ S. Holm and R. Sinkus, "A unifying fractional wave equation for compressional and shear waves," J. Acoust. Soc. Am. 127, 542-548 (2010).

${ }^{20} \mathrm{~F}$. Jarad, T. Abdeljawad, and D. Baleanu, “Fractional variational principles with delay within Caputo derivatives," Rep. Math. Phys. 65(1), 17-28 (2010).

${ }^{21}$ M. Klimek, "Lagrangean and Hamiltonian fractional sequential mechanics," Czech. J. Phys. 52(11), 1247-1253 (2002).

${ }^{22}$ L. Machado, L. Abrunheiro, and N. Martins, "Variational and optimal control approaches for the second-order Herglotz problem on spheres," J. Optim. Theory Appl. 182(3), 965-983 (2019).

${ }^{23}$ A. B. Malinowska, T. Odzijewicz, and D. F. M. Torres, Advanced Methods in the Fractional Calculus of Variations, Springer Briefs in Applied Sciences and Technology (Springer, Cham, 2015).

${ }^{24}$ A. B. Malinowska and D. F. M. Torres, "Generalized natural boundary conditions for fractional variational problems in terms of the Caputo derivative," Comput. Math. Appl. 59, 3110-3116 (2010).

${ }^{25}$ A. B. Malinowska and D. F. M. Torres, Introduction to the Fractional Calculus of Variations (Imperial College Press, London, 2012).

${ }^{26} \mathrm{~N}$. Martins, "A non-standard class of variational problems of Herglotz type" (submitted).

${ }^{27} \mathrm{~J}$. C. Orum, R. T. Hudspeth, W. Black, and R. B. Guenther, "Extension of the Herglotz algorithm to nonautonomous canonical transformations," SIAM Rev. 42(1), 83-90 (2000).

${ }^{28}$ C. M. A. Pinto and A. R. M. Carvalho, "Fractional order model for HIV dynamics," J. Comput. Appl. Math. 312, 240-256 (2017).

${ }^{29}$ F. Riewe, "Nonconservative Lagrangian and Hamiltonian mechanics," Phys. Rev. E 53(2), 1890-1899 (1996). 
${ }^{30}$ F. Riewe, "Mechanics with fractional derivatives," Phys. Rev. E 55(3), 3581-3592 (1997).

${ }^{31}$ A. A. Kilbas, H. M. Srivastava, and J. J. Trujillo, Theory and Applications of Fractional Differential Equations, North-Holland Mathematics Studies Vol. 204 (Elsevier Science B.V., Amsterdam, 2006).

${ }^{32}$ S. G. Samko, A. A. Kilbas, and O. I. Marichev, Fractional Integrals and Derivatives (Gordon and Breach, Yverdon, 1993) (translated from the 1987 Russian original).

${ }^{33}$ S. P. S. Santos, N. Martins, and D. F. M. Torres, "Higher-order variational problems of Herglotz type," Vietnam. J. Math. 42(4), 409-419 (2014).

${ }^{34}$ S. P. S. Santos, N. Martins, and D. F. M. Torres, "Variational problems of Herglotz type with time delay: Dubois-Reymond condition and Noether's first theorem," Discrete Contin. Dyn. Syst. 35(9), 4593-4610 (2015).

${ }^{35}$ S. P. S. Santos, N. Martins, and D. F. M. Torres, "Noether's theorem for higher-order variational problems of Herglotz type," in 10th AIMS Conference on Dynamical Systems, Dynamical Systems, Differential Equations and Applications (AIMS Proceedings) (AIMS, 2015), Vol. 2015, pp. 990-999.

${ }^{36} \mathrm{~T}$. Škovránek, I. Podlubny, and I. Petráš, “Modeling of the national economies in state-space: A fractional calculus approach," Econ. Modell. 29(4), 1322-1327 (2012).

${ }^{37}$ D. Tavares, R. Almeida, and D. F. M. Torres, “Fractional Herglotz variational problems of variable order,” Discrete Contin. Dyn. Syst., Ser. S 11(1), 143-154 (2018).

${ }^{38}$ Y. Zhang and X. Tian, "Conservation laws of nonconservative nonholonomic system based on Herglotz variational problem," Phys. Lett. A 383, 691-696 (2019).

${ }^{39}$ A. Zinober and S. Sufahani, "A non-standard optimal control problem arising in an economics application," Pesqui. Operacional 33(1), 63-71 (2013). 